\title{
Some Places Where I Spent Some Time
}

\section{Patrick Macklin}

Head of Department of Interior Design, The Glasgow School of Art, Glasgow, Scotland The Glasgow School of Art, 167 Renfrew Street, Glasgow G3 6RQ

email: p.macklin@gsa.ac.uk

telephone: 00 +44 (0)141 3534500 


\section{Some Places Where I Spent Some Time}

The point of departure is a single photograph. Taken by one of the original residents of a group of mid $20^{\text {th }}$ century tower block, located in the North Woodside area of Glasgow, it captures a place in transition, observed from the vantage point of one of its most recent additions, and provides context for the series of interior cameos that follow.

The stone tenements, once visible from the balcony, were distributed evenly throughout the city. They were clustered intact, in blocks stacked in a neat gridded arrangement as far as the eye could see, their deceptive order punctuated by public spaces and parkland, by glassworks, churches, foundries, mechanics yards, drying greens and chemical plants; by tight little stars of converging streets supporting fulcrums of activity. They predominantly contained peoples' homes, but along their perimeter were strung shops, offices, dentists, doctors' surgeries, pubs, hairdressers, dance halls, barbers, tailors, bookies, even a menagerie. The uniformity of their massive and modest construction concealed much. In some instances, once across the threshold, entry to the close (the semi-public stairwells) offered a palpable contrast between street and seclusion. Within were secreted highly decorated corridors and landings, punctuated by painted, colored, stained and etched glass which was festooned with organic representational and geometric abstracted motifs: fritillaries, lozenges and larks; quatrefoils, serpents and nonagons; press-moulded, brightly glazed ceramic tiles emblazoned with flowers and birds, in deep turquoise and dark mustard; mazy lines wending horizontally into distance and dimness; uniquely formed cast-iron stair spindles; decorated door mouldings and elaborate timber work all reaching a crescendo in the oculi, cupolas and complex, dizzying sweeps and turns of the stairways themselves. Sounds reflected from these surfaces, mimicking the scenes they portrayed, reverberations of brass against a lock mechanism; echoes of footsteps on a stair; chalk scraping out motifs resembling 
rangoli and muffled voices in transit. Further away from the streets, and insulated by the close itself, was the promise of gilding, ornamental plasterwork, friezes, ceiling roses, decorated fanlights, filigree and fretwork potentially nested in the apartments that residents and visitors alike would be irresistibly drawn towards, through this vortex.

The scene is very different now, since the neighborhood was sliced in half. The district sits under great arcs of billowing dust and smoke, where lengthy snaking trails of shattered glass glisten from beneath a fine skin of soot. Almost all of the neat rows of buildings lie eviscerated. Their contents scattered. Adjacent to these empty shells are vast mounds of smouldering timber and rubble. Shadows of filth sit in jarring contrast to the traces of pigment left behind by former occupants, that have come to demarcate the location of former kitchens and sculleries, where individual rectangles of carefully applied coloured washes accentuate great, black, iron ranges rendered frozen, silent and suspended in the air. Spectral and indelibly drawn charcoal staircases, doorways and window frames rise like ghostly apparitions, remnants of prior occupation. Wallpapers in areas within, previously protected from sunlight and grease, behind drapes and doors, are revealed in their unbleached original brilliance. Isolated chimneystacks occasionally rock in the breeze. The scene oscillates between the finality of the blow-down at PruittIgoe and the ellipsis of Gordon Matta-Clark's incisions at les Halles, but is so much later than the intervention of the former, and so much earlier than the provocation of the latter. In a sense it is simultaneously pre and post 'the modern', everything is back-tofront, asynchronous, predictive, predictable, out of time, and the future is next door. The apartment is an eleventh floor duplex housed in a multi-dwelling unit, a high density building, a slab block, one in a row of three clustered together, identical, like dominos. It is new and is made of concrete and steel. Its construction is simple, and was quick to assemble, consisting of interconnected panels stacked in a system, like 
building blocks. It has a decorative skin of fist-sized pebbles serving as a defence against the wind, and the rain, and the ice. This façade is punctuated with balconies and verandas. It is grey. Inside a wheeled table sits at the edge of the room in close proximity to the kitchen. Six players are perched around it, with silver sixpence wagers scattered constellation-like across its surface. They're playing Rummy with two decks, the edges of both so dulled that they sit at double their original height on the green baize, each stack of cards is as grey as the tower. A fug emanates from this point of focus; it comes mainly from un-tipped cigarettes and is buffeted by steam which ripples from the spout of a double handled bashed metal teapot. The players are first and second generation immigrants, who’ve been meeting like this on Sunday evenings every week for years. The players are the same, the table is the same, the cards are too, as is the twin-handled teapot, but the room is quite different, it is cozier, but starker, the ceiling is much lower. The furnishings, lamps fabrics and other paraphernalia of domesticity sit in contrast against its modest proportions and clean lines. It is clearly unadorned, an austerity partly softened by a face off between a portrait of JFK on one wall and Pope Paul VI on the other. There is a silent mouth organ on the writing bureau and an accordion rests on the plinth beside the two-bar electric fire-it seldom needs to be switched on.

Suburbia, a former shop, possibly a grocers, that has undergone a change of use, transformed, as if by magic, into a cottage. It sat at the end of a row, topped with a thick ribbon of slatted timber fascia where, presumably, once-upon-a-time, some signage was located. Made of red brick, in an untypical form for the suburbs, not quite a terrace, with more visible construction than render, it was penned into a strip of land at the bottom of a hill adjacent to the edge of a copse. A nested, hollowed-out space close to a seasonal stream and shielded from the sun for most of the year, but in early April the 
first rays of spring would illuminate the wall in the rooms at the back of the house, like the winter solstice dawn at Newgrange, a moment met with much ritualistic revelry. It was simply decorated with deep green metal window frames buckled with condensation and the intrusiveness of frost. Walls were ecclesiastical purple, chartreuse and tangerine softened under artificial light. It was often dingy during the day, and there were things everywhere, from tomes to tools to toys. In the darkness of the hallway hung a darkcolored, standard-issue corporation transport workers uniform, in heavy drill fabric, laced with the scent of cigarettes. Three pieces of equipment dominated that space, a Dansette record player, a Singer sewing machine and a double, rigid-hooded Eugene brand hair dryer. The former rested on the floor, surrounded by heaps of vinyl, with selections stacked in cylinders on the centre spindle in short, curated, visible playlists. The brightly hued labels of then dominant record companies appearing momentarily before being topped-out by the subsequent branding of the next disc. The player itself looked like a piece of luggage finished in petrol blue and white canvas with a white leatherette carry strap which spoke of portability and improvisation. The Singer, a relic, in ebony and gilded with flowers and curlicues, clattered into the night, staccato and productive, creating dressings for the windows and decorative enhancements for beds and sofas. The dryers formed the centrepiece of an improvised hair salon and looked like the engines of a B52. When spinning they helped disperse the acrid scent of ammonia from perm-lotion and the fruity smell of setting-lotion, so that their vapours would waft through the place on Friday afternoons, a day defined by this perfumed air and the sound of women chatting and talking over the drone of the whirring turbines.

Rewinding by almost a decade prior to now, to what appears to be the lowceilinged basement of another converted building, this time a compact, blocky venue that sits in splendid isolation by the side of a road. Within we see a band, a four-piece, 
whose drummer is atypically central and front of stage. The event appears cultish and rarified. They play against a backdrop of surfaces festooned in layers of stickers, a palimpsest of posters and flyers. The transparent acrylic drum-kit is tight to camera, which pans slowly, laterally across the room, back and forth, in a single languid shot. The top left of the frame is dominated by the view of a quadrant of a crash cymbal, its surface hit by spotlights, the sheen of the metal intermittently and incrementally shifting from cerulean through to arctic blue. The brass of the instrument annexes a large proportion of the screen and sits close to the drummer, framing his face. He is wearing a t-shirt with a Medusa like illustration on the front, she has her arm raised in a beatific pose with palm outstretched, his are wrapped around hickory drumsticks with which he delivers a mesmerising pulse that underpins the performance. He wears hearing protectors in a bright dayglo pink that echo the colour of the glimmering diodes on stacks of amps that form a temporary wall at the rear of the room, intense chromatic points of light floating against the blackness. The room is explored in a remote, comfortable and detached way, through pointing and clicking. The denuded nature of the experience is a provocation, lacking as it does, the certainty and authenticity of being there, it instead triggers reflection and dredges memory. Even relayed through micro-speakers of a laptop the nature and quality of the chamber clearly contributes to the intensity of the event, it's boxy enclosure at once contains and distributes sound and energy, it resonates atmosphere, even though - in spite of having the best view in the house - we only ever hear the audience. A vicarious experience, one that can be paused, re-cued and replayed, perhaps forever, but it can never happen again... not like this.

A brief journey through some spaces where I spent some time began with the decoding of an environment that had been captured, in an abbreviated way, in a single image, a photographic print, effectively a one-off, an analogue and outward looking 
document, it concluded with speculations on the possible spatial components of an online moving image, endlessly reproducible and in a sense indestructible, digital and self-contained. Both artefacts prompt consideration on the ways that matters of inhabitation, decoration, enclosure, intimacy and interaction are and might be communicated, and underlined how such clusters of crystals and pixels depend on augmentation when dealing with the more nebulous aspects of place.

Figure 1. View south over North Woodside, Glasgow circa 1967. Photographer unknown. 